PROCEEDINGS OF THE

AMERICAN MATHEMATICAL SOCIETY

Volume 133, Number 7 , Pages 1907-1915

S 0002-9939(05)07745-2

Article electronically published on January 21, 2005

\title{
VANISHING AND BASES FOR COHOMOLOGY OF PARTIALLY TRIVIAL LOCAL SYSTEMS ON HYPERPLANE ARRANGEMENTS
}

\author{
YUKIHITO KAWAHARA
}

(Communicated by Paul Goerss)

\begin{abstract}
In this paper we prove a vanishing theorem and construct bases for the cohomology of partially trivial local systems on complements of hyperplane arrangements. As a result, we obtain a non-resonance condition for partially trivial local systems.
\end{abstract}

\section{INTRODUCTION}

Let $\mathcal{A}$ be an arrangement of hyperplanes in $\mathbb{C}^{\ell}$, with complement $M=M(\mathcal{A})=$ $\mathbb{C}^{\ell} \backslash \bigcup_{H \in \mathcal{A}} H$. For a complex weight system $\lambda=\left(\lambda_{H}\right)_{H \in \mathcal{A}}$, we have a rank one local system $\mathcal{L}_{\lambda}$ on $M$ whose monodromy around the hyperplane $H$ is $\exp \left(-2 \pi \sqrt{-1} \lambda_{H}\right)$. For a hyperplane $H$ defined by a degree one polynomial $\alpha_{H}$, let $\omega_{H}=d \log \alpha_{H}=$ $d \alpha_{H} / \alpha_{H}$. Denote the sheaves of holomorphic $p$-forms on $M$ by $\Omega_{M}^{p}$ with $\Omega_{M}^{0}=\mathcal{O}_{M}$ and denote $\Omega^{p}(M)=\Gamma\left(M, \Omega_{M}^{p}\right)$. Define

$$
\omega_{\lambda}=\sum_{H \in \mathcal{A}} \lambda_{H} \omega_{H}, \quad \nabla_{\lambda}=d+\omega_{\lambda} \wedge
$$

where $d$ is the ordinary differential. The twisted de Rham theorem

$$
H^{p}\left(M, \mathcal{L}_{\lambda}\right) \simeq H^{p}\left(\Omega(M), \nabla_{\lambda}\right)
$$

is well known $([\mathrm{De}])$. In general, we know $H^{p}\left(M, \mathcal{L}_{\lambda}\right)=0$ for $p>\ell$. Let $k$ be a weight of $\mathcal{A}$ such that all $k_{H}$ 's are integers. The multiplication by the non-zero holomorphic function $\prod_{H \in \mathcal{A}} \alpha_{H}^{-k_{H}}$ on $M(\mathcal{A})$ induces an isomorphism of twisted de Rham complexes: $\left(\Omega(M), \nabla_{\lambda}\right) \simeq\left(\Omega(M), \nabla_{\lambda+k}\right)$. Namely, weights $\lambda$ and $\lambda+k$ give rise to the same local system (see [OT2, 2.1]). Since the local system $\mathcal{L}_{k}$ is equivalent to the constant sheaf $\mathbb{C}$, the local system cohomology is the usual cohomology $H^{*}(M, \mathbb{C})$. A weight $k$ is said to be trivial.

The local system cohomology $H^{*}\left(M, \mathcal{L}_{\lambda}\right)$, or the twisted de Rham cohomology, is an important subject in the Aomoto-Gelfand multivariable theory of hypergeometric functions [AK, Gel, OT2] and appears in various areas: the representation theory of Lie algebras and quantum groups Va]; the Knizhnik-Zamolodchikov differential equation in conformal field theory [EFK]. In order to compute the local

Received by the editors November 7, 2003 and, in revised form, February 26, 2004.

2000 Mathematics Subject Classification. Primary 14F40; Secondary 32S22, 55N25.

Key words and phrases. Hyperplane arrangement, local system, twisted de Rham cohomology.

(C)2005 American Mathematical Society Reverts to public domain 28 years from publication 
system cohomology, there are several methods: algebraic geometry, stratified Morse theory, combinatorics and so on.

We recall the vanishing theorem [ESV] STV, Yu1]. We use the notation of OT, OT2. Let $\mathbb{P}^{\ell}$ be the complex projective space, which is a compactification of $\mathbb{C}^{\ell}$ with the infinite hyperplane $\bar{H}_{\infty}$. The projective closure $\mathcal{A}_{\infty}$ of $\mathcal{A}$ is defined by $\mathcal{A}_{\infty}=\{\bar{H} \mid H \in \mathcal{A}\} \cup\left\{\bar{H}_{\infty}\right\}$, where $\bar{H}$ is the projective closure of $H$. Let $\lambda_{\infty}=-\sum_{H \in \mathcal{A}} \lambda_{H}$ be the weight of $\bar{H}_{\infty}$.

The intersection poset $L=L(\mathcal{A})$ of an (affine or projective) arrangement $\mathcal{A}$ is the set of nonempty intersections of elements of $\mathcal{A}$ with a partial order defined by reverse inclusion. We call an element of $L$ an edge of $\mathcal{A}$. The rank $r(X)$ of $X \in L$ is defined by $r(X)=\operatorname{codim}(X)$. The $\operatorname{rank} r(\mathcal{A})$ of $\mathcal{A}$ is the maximal rank of any edges of $\mathcal{A}$, which is the maximal number of linearly independent hyperplanes of $\mathcal{A}$ with nonempty intersection. For $X \in L(\mathcal{A})$, define

$$
\mathcal{A}_{X}=\{H \in \mathcal{A} \mid X \subset H\}, \quad \lambda_{X}=\sum_{H \in \mathcal{A}, X \subset H} \lambda_{H} .
$$

Let $\mathcal{C}$ be a central arrangement with center $\bigcap_{H \in \mathcal{C}} H \neq \emptyset$. We call $\mathcal{C}$ decomposable if there exist nonempty subarrangements $\mathcal{C}_{1}$ and $\mathcal{C}_{2}$ so that $\mathcal{C}=\mathcal{C}_{1} \cup \mathcal{C}_{2}$ is a disjoint union and after a linear coordinate change the defining polynomials for $\mathcal{C}_{1}$ and $\mathcal{C}_{2}$ have no common variables. An edge $X \in L(\mathcal{A})$ is called dense in $\mathcal{A}$ if the central arrangement $\mathcal{A}_{X}$ is not decomposable. Denote the set of all dense edges in $\mathcal{A}$ by $\mathrm{D}(\mathcal{A})$. Note that all hyperplanes of $\mathcal{A}$ are dense edges in $\mathcal{A}$.

Theorem 1.1 (ESV, STV, Yu1]). Let $\mathcal{A}$ be an affine arrangement of hyperplanes. If a weight $\lambda$ of $\mathcal{A}$ satisfies the condition:

$$
\lambda_{X} \notin \mathbb{Z}_{\geq 0} \quad \text { for every } X \in \mathrm{D}\left(\mathcal{A}_{\infty}\right),
$$

then we have

$$
H^{p}\left(M(\mathcal{A}), \mathcal{L}_{\lambda}\right)=0, \quad \text { for } p \neq r(\mathcal{A}) .
$$

This theorem says that a weight $\lambda$ satisfying (Mon) is non-resonant in the sense of [T2, 6.5]. In general, non-resonant weights give rise to a local system with vanishing cohomology in all dimensions except the top dimension. In the case of non-resonant weights, much is known (see OT2]).

For example, a basis of $H^{*}\left(M, \mathcal{L}_{\lambda}\right)$ is constructed by [FT] as follows. Let $\mathcal{A}=$ $\left\{H_{1}, H_{2}, \ldots, H_{n}\right\}$ be an arrangement with a linear order: $H_{i} \prec H_{j}$ if $i<j$. An independent set is a central subarrangement whose rank is equal to cardinality. A dependent set is a central subarrangement whose rank is less than cardinality. A base (or frame) is an inclusion-maximal independent set. Every base has cardinality $r=r(\mathcal{A})$. A circuit is an inclusion-minimal dependent set. A broken circuit is a subset $S$ for which there exists $H \prec \min (S)$ such that $\{H\} \cup S$ is a circuit. An nbc set is a non-empty subset of $\mathcal{A}$ that has nonempty intersection and contains no broken circuits. An nbc-base is a base which is an nbc set. The collection of nbc sets is a simplicial complex, called the nbc complex of $\mathcal{A}$. A base $B$ is called a $\beta \mathbf{n b c}$-base if $B$ is an nbc-base and, for every $H \in B$, there exists $H^{\prime} \prec H$ in $\mathcal{A}$ such that $(B \backslash\{H\}) \cup\left\{H^{\prime}\right\}$ is a base. Denote by $\beta \operatorname{nbc}(\mathcal{A})$ the set of $\beta$ nbc-bases. Let $\lambda$ be a weight of $\mathcal{A}$. For $X \in L(\mathcal{A})$ define

$$
\omega_{\lambda}(X)=\sum_{H \in \mathcal{A}_{X}} \lambda_{H} \omega_{H}
$$


For a base $B=\left\{H_{i_{1}}, H_{i_{2}}, \ldots, H_{i_{r}}\right\}$ with $i_{1}<i_{2}<\cdots<i_{r}$, define a flag $\xi(B)=$ $\left(X_{1} \subset X_{2} \subset \cdots \subset X_{r}\right)$ where $X_{p}=\bigcap_{k=p}^{r} H_{i_{k}}$, and define a logarithmic form

$$
\zeta(B)=\omega_{\lambda}\left(X_{1}\right) \wedge \cdots \wedge \omega_{\lambda}\left(X_{r}\right) .
$$

Theorem $1.2([\mathrm{FT}])$. Let $\mathcal{A}$ be an affine arrangement of hyperplanes and let $\lambda$ be its weight satisfying the condition (Mon). Then the set

$$
\{\zeta(B) \mid B \in \beta \mathbf{n b c}(\mathcal{A})\}
$$

is a basis of $H^{r(\mathcal{A})}\left(M(\mathcal{A}), \nabla_{\lambda}\right)$.

We can find many works and applications on the subject of calculations of the local system cohomology for any weights or resonant weights. The upper and lower bound of dimensions of the local system cohomology were studied in [Co1, CO1, LY]. The characteristic variety is the subvariety of the torus consisting of local systems for which the cohomology does not vanish [Li] [CS]. The characteristic varieties are deeply related to the cohomology support loci $\mathrm{Ar}$ ] and the resonance varieties [Fa, Yu2]. The Gauss-Manin connection on the local system cohomology is an important subject [Te, CO2] (see [OT2]).

The condition (Mon) demands that $\lambda_{H} \notin \mathbb{Z}_{>0}$ for $H \in \mathcal{A}$. In this paper, we remove this and then we treat a partially trivial weight $\lambda\left(\lambda_{H} \in \mathbb{Z}\right.$ for some $\left.H \in \mathcal{A}\right)$. For some partially trivial weight $\lambda$ which does not satisfy (Mon), we obtain a vanishing theorem and a basis for $H^{*}\left(M(\mathcal{A}), \mathcal{L}_{\lambda}\right)$, by using the above results and a long exact sequence for a triple. As a result, we obtain a condition on the weight $\lambda$ which is an extension of (Mon) and insures that the local system $\mathcal{L}_{\lambda}$ is non-resonant.

I would like to thank Professor Hiroaki Terao for many helpful suggestions and valuable discussions.

\section{Results}

Let $\mathcal{P}$ be an arrangement of hyperplanes in the complex projective space $\mathbb{P}^{\ell}$. For $H_{0} \in \mathcal{P}$, let $\mathbf{a f}_{H_{0}}(\mathcal{P})$ denote the affine arrangement $\left\{H \backslash H_{0}: H \in \mathcal{P}\right\}$ in $\mathbb{C}^{\ell}=\mathbb{P}^{\ell} \backslash H_{0}$. The rank of $\mathbf{a f}_{H_{0}}(\mathcal{P})$, denoted by $r_{\text {af }}(\mathcal{P})$, does not depend on the choice of $H_{0}$. Note that $r_{\text {af }}\left(\mathcal{A}_{\infty}\right)=r(\mathcal{A})$ for an affine arrangement $\mathcal{A}$.

Let $\mathcal{A}$ be an arrangement and let $\lambda$ be a weight of $\mathcal{A}$. By adding a trivial weight if necessary, we can assume that $\lambda_{H} \notin \mathbb{Z}_{\geq 0}$ or $\lambda_{H}=0$, that is, $\lambda_{H} \notin \mathbb{Z}_{>0}$, for $H \in \mathcal{A}$. Otherwise, it is possible to assume that $\lambda_{H} \notin \mathbb{Z}$ or $\lambda_{H}=0$ for $H \in \mathcal{A}$. We define a decomposition $\mathcal{A}=\mathcal{G}(\mathcal{A}, \lambda) \cup \mathcal{N}(\mathcal{A}, \lambda)$ of $\mathcal{A}$ by

$$
\mathcal{G}=\mathcal{G}(\mathcal{A}, \lambda)=\left\{H \in \mathcal{A} \mid \lambda_{H} \notin \mathbb{Z}_{\geq 0}\right\}
$$

and

$$
\mathcal{N}=\mathcal{N}(\mathcal{A}, \lambda)=\left\{H \in \mathcal{A} \mid \lambda_{H}=0\right\} .
$$

A weight $\lambda=\lambda(\mathcal{A})=\left(\lambda_{H}\right)_{H \in \mathcal{A}}$ of $\mathcal{A}$ induces a weight of a subarrangement $\mathcal{B}$ of $\mathcal{A}: \lambda(\mathcal{B})=\left(\lambda_{H}\right)_{H \in \mathcal{B}}$. Thus, the subarrangements $\mathcal{G}$ and $\mathcal{N}$ have weights $\lambda(\mathcal{G})$ and $\lambda(\mathcal{N})$ induced by $\lambda(\mathcal{A})$, respectively. Note that $\lambda(\mathcal{N})$ is trivial.

The first result in this paper is a generalization of Theorem 1.1 for partially trivial local systems, as follows.

Theorem 2.1. Let $\mathcal{A}$ be an affine arrangement and let $\lambda$ be its weight such that $\lambda_{H} \notin \mathbb{Z}_{>0}$ for $H \in \mathcal{A}$. If the weight $\lambda$ satisfies the condition

$\left(\right.$ Mon*) $\quad \lambda_{X} \notin \mathbb{Z}_{\geq 0} \quad$ for every $X \in \mathrm{D}\left(\mathcal{A}_{\infty}\right)$ with $\left(\mathcal{A}_{\infty}\right)_{X} \cap \mathcal{G}\left(\mathcal{A}_{\infty}, \lambda\right) \neq \emptyset$, 
then we have

$$
H^{k}\left(M(\mathcal{A}), \mathcal{L}_{\lambda}\right)=0, \quad \text { for } k<r,
$$

where $r=r_{\mathbf{a f}}\left(\mathcal{G}\left(\mathcal{A}_{\infty}, \lambda\right)\right)$.

Remark 2.2. If $\lambda$ is trivial, then $r=0$. If $\lambda$ is non-trivial, then $r>0$ and $H^{0}\left(M(\mathcal{A}), \mathcal{L}_{\lambda}\right)=0$. When $\mathcal{A}_{\infty}=\mathcal{G}\left(\mathcal{A}_{\infty}, \lambda\right)$, this is Theorem 1.1. When $r=$ $r(\mathcal{A})=r_{\text {af }}\left(\mathcal{G}\left(\mathcal{A}_{\infty}, \lambda\right)\right)$, we have

$$
H^{k}\left(M(\mathcal{A}), \mathcal{L}_{\lambda}\right)=0, \quad \text { for } k \neq r .
$$

Consequently, a weight $\lambda$ of $\mathcal{A}$ satisfying (Mon*) and $r(\mathcal{A})=r_{\text {af }}\left(\mathcal{G}\left(\mathcal{A}_{\infty}, \lambda\right)\right)$, is non-resonant in the sense of [OT2, 6.5].

Next we shall construct a basis for partially trivial local systems. Suppose $\lambda_{\infty} \notin$ $\mathbb{Z}$. We modify the definition of $\omega_{\lambda}(X)$ from section 1$]$ as follows:

$$
\omega_{\lambda}(X)=\sum_{H \in \mathcal{A}_{X}, \lambda_{H} \notin \mathbb{Z}_{\geq 0}} \lambda_{H} \omega_{H}+\sum_{H \in \mathcal{A}_{X}, \lambda_{H} \in \mathbb{Z}_{\geq 0}} \omega_{H} .
$$

Theorem 2.3. Under the assumption in Theorem 2.1, we moreover assume that $\lambda_{\infty} \notin \mathbb{Z}$ and $r=r(\mathcal{A})=r_{\text {af }}\left(\mathcal{G}\left(\mathcal{A}_{\infty}, \lambda\right)\right)$. Then the set

$$
\{\zeta(B) \mid B \in \beta \mathbf{n b c}(\mathcal{A})\}
$$

is a basis of $H^{r}\left(M(\mathcal{A}), \mathcal{L}_{\lambda}\right)$.

\section{Proofs}

A triple $\left(\mathcal{A}, \mathcal{A}^{\prime}, \mathcal{A}^{\prime \prime}\right)_{H}$ of arrangements with distinguished hyperplane $H \in \mathcal{A}$ is defined by $\mathcal{A}^{\prime}=\mathcal{A} \backslash\{H\}$ and $\mathcal{A}^{\prime \prime}=\mathcal{A}^{H}$. A weight $\lambda$ of $\mathcal{A}$ induces a weight of a subarrangement $\mathcal{A}^{\prime}$ and a weight of $\mathcal{A}^{\prime \prime}$ defined by

$$
\lambda_{H^{\prime \prime}}=\sum_{I \in \mathcal{A}, I \supset H^{\prime \prime}, I \neq H} \lambda_{I}
$$

for $H^{\prime \prime} \in \mathcal{A}^{\prime \prime}$.

Let $H$ be a hyperplane in $\mathbb{C}^{\ell}$. A tubular neighborhood $T_{H}$ of $H$ in $\mathbb{C}^{\ell}$ is a trivial bundle over $H$ with fiber $F=\mathbb{C}$. The complement of the zero section is denoted by $T_{H}^{*}=T_{H} \backslash H$. Since $H$ is contractible, for any fiber $F_{0}$ of $T_{H}^{*}$, we have

$$
H^{1}\left(F_{0}\right)=H^{1}\left(T_{H}^{*}\right)=\mathbb{C}\left\{\omega_{H}\right\} .
$$

Theorem $3.1(\mathrm{Co} 2, \mathrm{Ka}])$. Let $\left(\mathcal{A}, \mathcal{A}^{\prime}, \mathcal{A}^{\prime \prime}\right)_{H}$ be a triple of arrangements with distinguished hyperplane $H \in \mathcal{A}$. Let $\lambda$ be a weight of $\mathcal{A}$ with $\lambda_{H}=0$. Then we have a long exact sequence:

$$
\begin{aligned}
\cdots & \rightarrow H^{k}\left(M\left(\mathcal{A}^{\prime}\right), \mathcal{L}_{\lambda}\right) \rightarrow H^{k}\left(M(\mathcal{A}), \mathcal{L}_{\lambda}\right) \rightarrow H^{k-1}\left(M\left(\mathcal{A}^{\prime \prime}\right), \mathcal{L}_{\lambda}\right) \otimes H^{1}\left(T_{H}^{*}\right) \\
& \rightarrow H^{k+1}\left(M\left(\mathcal{A}^{\prime}\right), \mathcal{L}_{\lambda}\right) \rightarrow \cdots .
\end{aligned}
$$

Let $\left(\mathcal{C}, \mathcal{C}^{\prime}, \mathcal{C}^{\prime \prime}\right)_{H}$ be a triple of central arrangements with distinguished hyperplane $H$. We call $H$ a separator if the center $T(\mathcal{C})$ of $\mathcal{C}$ does not belong to $L\left(\mathcal{C}^{\prime}\right.$ ) (see OT, Definition 2.58]). In other words, we can set $\mathcal{C}=\mathcal{C}^{\prime} \times\{H\}$.

Lemma 3.2. Let $\left(\mathcal{C}, \mathcal{C}^{\prime}, \mathcal{C}^{\prime \prime}\right)_{H}$ be a triple of central arrangements with distinguished hyperplane $H$. If $H$ is a separator, then $\mathcal{C}$ is decomposable and we have

$$
\mathcal{C}^{\prime} \text { is decomposable } \Longleftrightarrow \mathcal{C}^{\prime \prime} \text { is decomposable. }
$$


If $H$ is not a separator, then we have

$$
\mathcal{C} \text { is decomposable } \Longleftrightarrow \mathcal{C}^{\prime} \text { and } \mathcal{C}^{\prime \prime} \text { are decomposable. }
$$

Proof. Assume that $H$ is a separator. Clearly, $\mathcal{C}$ is decomposable. Since $\mathcal{C}=$ $\mathcal{C}^{\prime} \times\{H\}$, we have $\mathcal{C}^{\prime \prime}=\mathcal{C}^{H}=\mathcal{C}^{\prime}$ and thus the first statement.

Assume that $H$ is not a separator. By OT2, Lemma 3.2.4.], if $\mathcal{C}^{\prime}$ and $\mathcal{C}^{\prime \prime}$ are decomposable, then $\mathcal{C}$ is decomposable. Suppose that $\mathcal{C}$ is decomposable with $\mathcal{C}=$ $\mathcal{C}_{1} \times \mathcal{C}_{2}$ and $H \in \mathcal{C}_{2}$. Since $H$ is not a separator, we have $\mathcal{C}_{2} \backslash\{H\} \neq \emptyset$. Then $\mathcal{C}^{\prime}=\mathcal{C}_{1} \times\left(\mathcal{C}_{2} \backslash\{H\}\right)$ is decomposable and $\mathcal{C}^{\prime \prime}=\mathcal{C}^{H}=\mathcal{C}_{1} \times \mathcal{C}_{2}^{H}$ is decomposable as well.

Proof of Theorem 2.1. Let $\lambda$ be a non-trivial weight of an affine arrangement $\mathcal{A}$. By changing the infinite hyperplane, if necessary, we can assume that $\lambda_{\infty} \notin \mathbb{Z}$. Since $\mathcal{G}\left(\mathcal{A}_{\infty}, \lambda\right)$ contains the infinite hyperplane, $\mathcal{N}\left(\mathcal{A}_{\infty}, \lambda\right)$ and $\mathcal{N}(\mathcal{A}, \lambda)$ have the same cardinality $s$. We shall prove this theorem using induction on $s$. When $s=0$, the condition (Mon*) is equivalent to the condition (Mon), and then it is true by Theorem 1.1 ([ESV, STV]). Note that, when $r(\mathcal{A})=1$, the result holds. Write $\mathcal{G}=\mathcal{G}(\mathcal{A}, \lambda), \mathcal{N}=\mathcal{N}(\mathcal{A}, \lambda)$ and $r=r(\mathcal{G})$.

Suppose that the statements are true for all weighted arrangements satisfying (Mon*) and $|\mathcal{N}|<s$. Assume that the weight $\lambda$ of $\mathcal{A}$ satisfies (Mon*) and $s=$ $|\mathcal{N}(\mathcal{A}, \lambda)|$. Fixing a hyperplane $H \in \mathcal{N}$, we take the triple $\left(\mathcal{A}, \mathcal{A}^{\prime}, \mathcal{A}^{\prime \prime}\right)_{H}$ with distinguished hyperplane $H$. Note that $\lambda_{H}=0$. Write $\mathcal{G}^{\prime}=\mathcal{G}\left(\mathcal{A}^{\prime}, \lambda\right), \mathcal{N}^{\prime}=$ $\mathcal{N}\left(\mathcal{A}^{\prime}, \lambda\right), r^{\prime}=r\left(\mathcal{G}^{\prime}\right)$ and $s^{\prime}=\left|\mathcal{N}\left(\mathcal{A}^{\prime}, \lambda\right)\right|$ for $\mathcal{A}^{\prime}$ and write $\mathcal{G}^{\prime \prime}=\mathcal{G}\left(\mathcal{A}^{\prime \prime}, \lambda\right), \mathcal{N}^{\prime \prime}=$ $\mathcal{N}\left(\mathcal{A}^{\prime \prime}, \lambda\right), r^{\prime \prime}=r\left(\mathcal{G}^{\prime \prime}\right)$ and $s^{\prime \prime}=\left|\mathcal{N}\left(\mathcal{A}^{\prime \prime}, \lambda\right)\right|$ for $\mathcal{A}^{\prime \prime}$.

First, we shall show that induced weights $\lambda$ of $\mathcal{A}^{\prime}$ and $\mathcal{A}^{\prime \prime}$ satisfy the condition (Mon*). By [0T2, Lemma 3.2.6] we have $D\left(\mathcal{A}_{\infty}^{\prime}\right) \subset D\left(\mathcal{A}_{\infty}\right)$. Since $\mathcal{G}\left(\mathcal{A}_{\infty}, \lambda\right)=$ $\mathcal{G}\left(\mathcal{A}_{\infty}^{\prime}, \lambda\right)$, the weight $\lambda$ of $\mathcal{A}^{\prime}$ satisfies (Mon*). Let $X^{\prime \prime} \in \mathrm{D}\left(\mathcal{A}_{\infty}^{\prime \prime}\right)$ be such that there exists $H \in \mathcal{A}_{\infty}^{\prime \prime}$ containing $X^{\prime \prime}$, whose weight $\lambda_{H}$ is not an integer. As $X^{\prime \prime} \in L\left(\mathcal{A}_{\infty}\right)$, we denote by $\mathcal{C}$ the central arrangement $\left(\mathcal{A}_{\infty}\right)_{X^{\prime \prime}}$ with center $X^{\prime \prime}$. Since $H \in \mathcal{C}$, we take the triple $\left(\mathcal{C}, \mathcal{C}^{\prime}, \mathcal{C}^{\prime \prime}\right)_{H}$ with distinguished hyperplane $H$, whose centers are $\left(X, X^{\prime}, X^{\prime \prime}\right)$ respectively. Since $\lambda_{H}=0$, we have

$$
\lambda_{X}=\lambda_{X^{\prime}}=\lambda_{X^{\prime \prime}}=\lambda_{\mathcal{C}}=\lambda_{\mathcal{C}^{\prime}}=\lambda_{\mathcal{C}^{\prime \prime}} .
$$

On the other hand, $\mathcal{C}^{\prime \prime}$ is not decomposable because $X^{\prime \prime} \in \mathrm{D}\left(\mathcal{A}_{\infty}^{\prime \prime}\right)$. By Lemma 3.2 we obtain the following. If $H$ is a separator, then $\mathcal{C}^{\prime}$ is not decomposable and hence $X^{\prime} \in \mathrm{D}\left(\mathcal{A}_{\infty}^{\prime}\right)$. We get $\lambda_{X^{\prime \prime}}=\lambda_{X^{\prime}} \notin \mathbb{Z}_{\geq 0}$. If $H$ is not a separator, then $\mathcal{C}$ is not decomposable and hence $X \in \mathrm{D}\left(\mathcal{A}_{\infty}\right)$. We get $\lambda_{X^{\prime \prime}}=\lambda_{X} \notin \mathbb{Z}_{\geq 0}$. Therefore, the weight $\lambda$ of $\mathcal{A}^{\prime \prime}$ satisfies (Mon $*$ ).

Secondly, we study $\mathcal{G}^{\prime}$ and $\mathcal{G}^{\prime \prime}$. Since $H \in \mathcal{N}$, it is clear that $\mathcal{G}^{\prime}=\mathcal{G}$ and then $r^{\prime}=r$. We shall show that $\mathcal{G}^{\prime \prime}$ is the restriction $\mathcal{G}^{H}$ of $\mathcal{G}$ to $H$. It is clear that $\mathcal{G}^{\prime \prime} \subset \mathcal{G}^{H}$. We take any $X \in \mathcal{G}^{H}$. If $\mathcal{G}_{X}=\left\{H_{1}, \ldots, H_{p}\right\}$, then $X=H_{1} \cap \cdots \cap H_{p} \cap H$. Since $X$ is a hyperplane in $\mathcal{G}^{H}$, we have $r(X)=2$ as the rank in $\mathcal{G}$. On the other hand, because $r(X) \geqq r\left(H_{1} \cap \cdots \cap H_{p}\right) \neq 1$, we have $r\left(H_{1} \cap \cdots \cap H_{p}\right)=2$. If $p=1$, then $X=H_{1} \cap H$ and we get $\lambda_{X}=\lambda_{H_{1}}+0 \notin \mathbb{Z}_{\geq 0}$. If $p>1$, then $X$ is dense and hence $\lambda_{X} \notin \mathbb{Z}_{\geq 0}$. Therefore, we have $\mathcal{G}^{\prime \prime}=\mathcal{G}^{H}$.

Finally, since $s^{\prime}<s$ and $s^{\prime \prime}<s$, the vanishing theorem holds for $\mathcal{A}^{\prime}$ and $\mathcal{A}^{\prime \prime}$ by inductive assumptions. Since $r^{\prime}=r$ and $r^{\prime \prime}$ is $r$ or $r-1$, we obtain

$$
H^{k}\left(M\left(\mathcal{A}^{\prime}\right), \mathcal{L}_{\lambda}\right)=0, H^{k-1}\left(M\left(\mathcal{A}^{\prime \prime}\right), \mathcal{L}_{\lambda}\right)=0, \quad \text { for } k<r .
$$


Therefore, the long exact sequence for the triple $\left(\mathcal{A}, \mathcal{A}^{\prime}, \mathcal{A}^{\prime \prime}\right)_{H}$ in Theorem $3.1 \mathrm{im}$ plies that

$$
H^{k}\left(M(\mathcal{A}), \mathcal{L}_{\lambda}\right)=0, \quad \text { for } k<r,
$$

and

$$
0 \rightarrow H^{r}\left(M(\mathcal{G}), \mathcal{L}_{\lambda}\right) \rightarrow H^{r}\left(M(\mathcal{A}), \mathcal{L}_{\lambda}\right)
$$

We obtain the following corollary of the proof of Theorem 2.1

Corollary 3.3. Under the assumption in Theorem 2.1, the inclusion map from $M(\mathcal{A})$ to $M\left(\mathcal{G}\left(\mathcal{A}_{\infty}, \lambda\right)\right.$ induces an injective map

$$
H^{r}\left(M\left(\mathcal{G}\left(\mathcal{A}_{\infty}, \lambda\right)\right), \mathcal{L}_{\lambda}\right) \rightarrow H^{r}\left(M(\mathcal{A}), \mathcal{L}_{\lambda}\right)
$$

where $M\left(\mathcal{G}\left(\mathcal{A}_{\infty}, \lambda\right)\right)$ is the complement of the projective arrangement $\mathcal{G}\left(\mathcal{A}_{\infty}, \lambda\right)$. In particular, if $H^{r}\left(M\left(\mathcal{G}\left(\mathcal{A}_{\infty}, \lambda\right)\right), \mathcal{L}_{\lambda}\right) \neq 0$, then $H^{r}\left(M(\mathcal{A}), \mathcal{L}_{\lambda}\right) \neq 0$.

Proof of Theorem 2.3. By $[\mathrm{FT}$, the space generated by $\{\zeta(B) \mid B \in \beta \mathbf{n b c}(\mathcal{A})\}$ is not dependent on a linear order in $\mathcal{A}$. We choose a linear order in $\mathcal{A}$ as follows : if $H_{i} \in \mathcal{G}(\mathcal{A}, \lambda)$ and $H_{j} \in \mathcal{N}(\mathcal{A}, \lambda)$, then $i<j$.

We use the same induction on $s$ in the proof of Theorem [2.1. When $s=0$, the condition $(\mathrm{Mon} *)$ is equivalent to the condition (Mon), and then Theorem 2.3 holds by Theorem $1.2[\overline{\mathrm{FT}}]$. Assume that $s>0$. We take the triple $\left(\mathcal{A}, \mathcal{A}^{\prime}, \mathcal{A}^{\prime \prime}\right)_{H_{n}}$, where $H_{n}$ is the last hyperplane. By the definition of the linear order, note that $H_{n} \in \mathcal{N}(\mathcal{A}, \lambda)$. Since $r=r(\mathcal{A})=r\left(\mathcal{A}^{\prime}\right)=r(\mathcal{G})$, we note that $r\left(\mathcal{G}^{\prime \prime}\right)=r\left(\mathcal{A}^{\prime \prime}\right)=r-1$. From Theorem 3.1 and Theorem [2.1, we have a short exact sequence:

$$
0 \rightarrow H^{r}\left(M\left(\mathcal{A}^{\prime}\right), \mathcal{L}_{\lambda}\right) \rightarrow H^{r}\left(M(\mathcal{A}), \mathcal{L}_{\lambda}\right) \rightarrow H^{r-1}\left(M\left(\mathcal{A}^{\prime \prime}\right), \mathcal{L}_{\lambda}\right) \otimes H^{1}\left(T_{H_{n}}^{*}\right) \rightarrow 0 .
$$

Using inductive assumptions, we have

$$
\left\{\zeta\left(B^{\prime}\right) \mid B^{\prime} \in \beta \mathbf{n b c}\left(\mathcal{A}^{\prime}\right)\right\} \quad \text { and } \quad\left\{\zeta\left(B^{\prime \prime}\right) \mid B^{\prime \prime} \in \beta \mathbf{n b c}\left(\mathcal{A}^{\prime \prime}\right)\right\}
$$

are bases of $H^{r}\left(M\left(\mathcal{A}^{\prime}\right), \mathcal{L}_{\lambda}\right)$ and $H^{r-1}\left(M\left(\mathcal{A}^{\prime \prime}\right), \mathcal{L}_{\lambda}\right)$, respectively. Define a map $\nu: L(\mathcal{A}) \backslash\left\{\mathbb{C}^{\ell}\right\} \rightarrow \mathcal{A}$ by $\nu X=\min \left(\mathcal{A}_{X}\right)$. Note that, for $H^{\prime \prime} \in \mathcal{A}^{\prime \prime}$, since $H_{n} \in \mathcal{A}_{H^{\prime \prime}}$ and $H_{n}$ is the largest hyperplane, we have $\nu H^{\prime \prime} \in \mathcal{A}^{\prime}$. For $S=\left(X_{1}, \ldots, X_{p}\right)$, write $\nu S=\left(\nu X_{1}, \ldots, \nu X_{p}\right)$. Due to [OT2, Theorem 5.3.2], we have a disjoint union

$$
\beta \mathbf{n b c}(\mathcal{A})=\beta \operatorname{nbc}\left(\mathcal{A}^{\prime}\right) \cup\left\{\left\{\nu B^{\prime \prime}, H_{n}\right\} \mid B^{\prime \prime} \in \beta \mathbf{n b c}\left(\mathcal{A}^{\prime \prime}\right)\right\} .
$$

Note that

$$
\zeta\left(\left\{\nu B^{\prime \prime}, H_{n}\right\}\right)=\zeta\left(B^{\prime \prime}\right) \wedge \omega_{H_{n}}
$$

(see [OT2, Lemma 6.3.5] for details). By the above short exact sequence, the set $\{\zeta(B) \mid B \in \beta \mathbf{n b c}(\mathcal{A})\}$ generates $H^{r}\left(M(\mathcal{A}), \mathcal{L}_{\lambda}\right)$.

By the vanishing theorem, we have

$$
\operatorname{dim} H^{r}\left(M(\mathcal{A}), \mathcal{L}_{\lambda}\right)=|\chi(\mathcal{A})|,
$$

where $\chi(\mathcal{A})$ is the Euler characteristic of $M(\mathcal{A})$. Moreover, we know that $|\chi(\mathcal{A})|$ is equal to the cardinality of $\beta \operatorname{nbc}(\mathcal{A})$, which is the beta invariant $\beta(\mathcal{A})$ defined in OT2, Section 3.3] (see also [STV]). Therefore, we obtain

$$
\operatorname{dim} H^{r}\left(M(\mathcal{A}), \mathcal{L}_{\lambda}\right)=|\beta \mathbf{n b c}(\mathcal{A})|=\beta(\mathcal{A}) .
$$

Note that $\beta(\mathcal{A})=\beta\left(\mathcal{A}^{\prime}\right)+\beta\left(\mathcal{A}^{\prime \prime}\right)$ (see [OT2, Proposition 3.3.3]).

As a result, the set $\{\zeta(B) \mid B \in \beta \operatorname{nbc}(\mathcal{A})\}$ is a basis of $H^{r}\left(M(\mathcal{A}), \mathcal{L}_{\lambda}\right)$. We have completed the proof. 


\section{EXAmples}

Let $\mathcal{A}=\left\{H_{1}, H_{2}, \ldots, H_{n}\right\}$ be an arrangement and $\lambda$ be its weight. In this section, we shall show examples of weights of the following types:

(A) $\lambda$ satisfies (Mon*) and does not satisfy (Mon), but there exists a trivial weight $k$ for which $\lambda+k$ satisfies (Mon).

(B) $\lambda$ satisfies (Mon*), and there is no trivial weight $k$ for which $\lambda+k$ satisfies (Mon).

Weights of type A essentially satisfy (Mon). Examples of weights of type B imply that Theorem 2.1 essentially extends the results of Theorem 1.1. Furthermore, there are occasions when we can compute the local system cohomology by the way of the proof of Theorem 2.1.

Define the subset $\mathrm{ND}(\lambda)$ of $\mathrm{D}\left(\mathcal{A}_{\infty}\right)$ by

$$
\mathrm{ND}(\lambda)=\left\{X \in \mathrm{D}\left(\mathcal{A}_{\infty}\right): \lambda_{X} \in \mathbb{Z}_{\geq 0}\right\} .
$$

So $\lambda$ satisfies (Mon) if and only if $\operatorname{ND}(\lambda)=\emptyset$. Let $h^{*}=h^{*}(\mathcal{A})=h^{*}(\mathcal{A}, \lambda)=$ $\operatorname{dim} H^{*}\left(M(\mathcal{A}), \mathcal{L}_{\lambda}\right)$, for short. Write $X_{i_{1} \cdots i_{s}}=\bar{H}_{i_{1}} \cap \cdots \cap \bar{H}_{i_{s}}, \lambda_{i}=\lambda_{H_{i}}$ and $\lambda_{i_{1} \cdots i_{s}}=\lambda_{X_{i_{1} \cdots i_{s}}}$. Note that $\sum_{1 \leq i<n} \lambda_{i}+\lambda_{\infty}=0$. The Selberg 2-arrangement $\mathcal{S}$ consists of $H_{1}: x=0, H_{2}: y=0, H_{3}: x=y, H_{4}: x=1$ and $H_{5}: y=1$ in $\mathbb{C}(x, y)$. We have

$$
\mathrm{D}\left(\mathcal{S}_{\infty}\right)=\left\{\bar{H}_{1}, \ldots, \bar{H}_{5}, \bar{H}_{\infty}, X_{123}, X_{345}, X_{14 \infty}, X_{25 \infty}\right\} .
$$

Example 4.1. Let $\mathcal{A}=\mathcal{S}$ and $\lambda=(0,0,1 / 4,1 / 4,1 / 4)$ with $\lambda_{\infty}=-3 / 4$. Since $\operatorname{ND}(\lambda)=\left\{\bar{H}_{1}, \bar{H}_{2}\right\}$, the weight $\lambda$ satisfies (Mon*) and thus we have $h^{1}=h^{0}=0$. But, for $k=(-1,-1,0,0,0)$, the weight $\lambda+k$ satisfies (Mon). Then $\lambda$ is of type A.

The weight $\lambda=(0,0,0,1 / 3,1 / 3)$ is of type A, as well. Note that $\operatorname{ND}(\lambda)=$ $\left\{\bar{H}_{1}, \bar{H}_{2}, \bar{H}_{3}, X_{123}\right\}$.

Example 4.2. Let $\mathcal{A}=\mathcal{S}$. The weight $\lambda=(0,2 / 3,2 / 3,1 / 3,-2)$ satisfies (Mon*). Then we have $h^{1}=h^{0}=0$. Let $\tilde{\lambda}=\lambda+(-1,0,0,0,0)$. Since $\tilde{\lambda}_{14 \infty}=0$, the integer shift $\tilde{\lambda}$ does not satisfy (Mon). By concrete computation, there is no trivial weight $k$ for which $\lambda+k$ satisfies (Mon). Therefore the weight $\lambda$ is of type B.

Example 4.3. Let $\mathcal{A}=\mathcal{S}$. The weight $\lambda=(0,0,1 / 3,0,0)$ is of type A. But, since $r_{\text {af }}\left(\mathcal{G}\left(\mathcal{A}_{\infty}, \lambda\right)\right)=1$, Theorem 2.1 says that $h^{0}=0$. On the other hand, we have $\lambda+(-1,-1,0,-1,-1)$ satisfies (Mon) and thus we know $h^{1}=h^{0}=0$. Otherwise, we can compute the local system cohomology by using the long exact sequence for a triple. Let $\mathcal{A}_{1}=\left\{H_{1}, H_{3}, H_{5}\right\}, \mathcal{A}_{2}=\mathcal{A}_{1} \cup\left\{H_{2}\right\}$ and $\mathcal{A}_{3}=\mathcal{A}_{2} \cup\left\{H_{4}\right\}=\mathcal{A}$ be subarrangements of $\mathcal{A}$. They have weights induced by $\lambda$. The weight $\lambda\left(\mathcal{A}_{1}\right)$ satisfies $($ Mon $*)$, and then $h^{1}\left(\mathcal{A}_{1}\right)=h^{0}\left(\mathcal{A}_{1}\right)=0$. For the triple $\left(\mathcal{A}_{2}, \mathcal{A}_{2}^{\prime}=\mathcal{A}_{1}, \mathcal{A}_{2}^{\prime \prime}\right)_{H_{2}}$, we have $H^{*}\left(M\left(\mathcal{A}_{2}^{\prime \prime}\right), \mathcal{L}_{\lambda}\right)=0$ and the long exact sequence for the triple induces $h^{1}\left(\mathcal{A}_{2}\right)=h^{0}\left(\mathcal{A}_{2}\right)=0$. Next, for the triple $\left(\mathcal{A}_{3}=\mathcal{A}, \mathcal{A}_{3}^{\prime}=\mathcal{A}_{2}, \mathcal{A}_{3}^{\prime \prime}\right)_{H_{4}}$, we have $h^{0}\left(\mathcal{A}_{3}^{\prime \prime}\right)=0$ and the long exact sequence for this triple induces $h^{1}(\mathcal{A})=h^{0}(\mathcal{A})=0$.

Example 4.4. Let $\mathcal{A}=\mathcal{S}$ and $\lambda=(1 / 3,0,0,0,0)$ with $\lambda_{\infty}=-1 / 3$. Since $\lambda_{14 \infty}=$ 0 , the weight $\lambda$ does not satisfy (Mon*) and there is no integer shift of $\lambda$ satisfying (Mon). But, we can compute the local system cohomology in the following way. Let $\mathcal{B}_{1}=\left\{H_{1}\right\}, \mathcal{B}_{2}=\left\{H_{2}, H_{5}\right\}, \mathcal{A}_{1}=\left\{H_{1}, H_{2}, H_{5}\right\}, \mathcal{A}_{2}=\mathcal{A}_{1} \cup\left\{H_{4}\right\}$ and $\mathcal{A}_{3}=$ $\mathcal{A}_{2} \cup\left\{H_{3}\right\}=\mathcal{A}$ be subarrangements of $\mathcal{A}$. They have weights induced by $\lambda$. Since 
essentially $\mathcal{B}_{1} \times \mathcal{B}_{2}=\mathcal{A}_{1}$, the twisted version of the Künneth formula and $h^{*}\left(\mathcal{B}_{1}\right)=$ 0 , we have $h^{*}\left(\mathcal{A}_{1}\right)=0$. By the long exact sequence for the triple $\left(\mathcal{A}_{2}, \mathcal{A}_{2}^{\prime}=\right.$ $\left.\mathcal{A}_{1}, \mathcal{A}_{2}^{\prime \prime}\right)_{H_{4}}$, since $\lambda\left(\mathcal{A}_{2}^{\prime \prime}\right)$ is trivial and $h^{1}\left(\mathcal{A}_{2}^{\prime \prime}\right)=2, h^{0}\left(\mathcal{A}_{2}^{\prime \prime}\right)=1$, we get $h^{2}\left(\mathcal{A}_{2}\right)=$ $2, h^{1}\left(\mathcal{A}_{2}\right)=1, h^{0}\left(\mathcal{A}_{2}\right)=0$. Finally, the long exact sequence for the triple $\left(\mathcal{A}_{3}=\right.$ $\left.\mathcal{A}, \mathcal{A}_{3}^{\prime}=\mathcal{A}_{2}, \mathcal{A}_{3}^{\prime \prime}\right)_{H_{3}}$ induces

$$
h^{2}(\mathcal{A}, \lambda)=3, h^{1}(\mathcal{A}, \lambda)=1, h^{0}(\mathcal{A}, \lambda)=0 .
$$

On the other hand, let $\mathcal{A}_{0}=\mathcal{S} \backslash\left\{H_{5}\right\}$ be the subarrangement of $\mathcal{A}$. The induced weight $\lambda\left(\mathcal{A}_{0}\right)$ satisfies $(\mathrm{Mon} *)$, and thus we have $h^{2}\left(\mathcal{A}_{0}\right)=1, h^{1}\left(\mathcal{A}_{0}\right)=h^{0}\left(\mathcal{A}_{0}\right)=0$. Note that $h^{0}\left(\mathcal{A}^{\prime \prime}\right)=1, h^{1}\left(\mathcal{A}^{\prime \prime}\right)=2$. In the long exact sequence for the triple $\left(\mathcal{A}, \mathcal{A}^{\prime}=\mathcal{A}_{0}, \mathcal{A}^{\prime \prime}\right)_{H_{5}}$, the coboundary map $H^{0}\left(M\left(\mathcal{A}^{\prime \prime}\right), \mathcal{L}_{\lambda}\right) \rightarrow H^{1}\left(M\left(\mathcal{A}^{\prime}\right), \mathcal{L}_{\lambda}\right)$ is an isomorphism. Therefore, in general, coboundary maps in the long exact sequence for a triple are not zero maps. In the case for a trivial weight, all coboundary maps are zero maps ([OT, Theorem 5.87]).

Example 4.5. Let $\mathcal{A}=\mathcal{S} \cup\left\{H_{6}\right\}$ defined by $H_{6}: x+y=1$. We have

$$
\mathrm{D}\left(\mathcal{A}_{\infty}\right)=\mathrm{D}\left(\mathcal{S}_{\infty}\right) \cup\left\{\bar{H}_{6}, X_{156}, X_{246}\right\} .
$$

It is easy to check the following:

(1) The weight $\lambda=(0,0,0,1 / 4,1 / 4,1 / 4)$ is of type $\mathrm{A}$.

(2) The weight $\lambda=(0,-2 / 3,4 / 3,-5 / 3,-2 / 3,4 / 3)$ is of type $\mathrm{B}$.

(3) The weight $\lambda=(0,2 / 3,1 / 2,1 / 2,-2,0)$ is of type $\mathrm{B}$.

\section{REFERENCES}

[Ar] Arapura, D., Geometry of cohomology support loci for local systems I, J. Alg. Geom. 6 (1997), 563-597. MR1487227 (98m:32043)

[AK] Aomoto, K. and Kita, M., Hypergeometric functions (in Japanese), Tokyo Springer, (1994).

[Co1] Cohen, D., Morse inequalities for arrangements, Adv. Math. 134 (1998), 43-45. MR:1612383 (98m:52017)

[Co2] Cohen, D., Triples of arrangements and local systems, Proc. Amer. Math. Soc. 130 (2002), no. 10, 3025-3031. MR 1908926 (2003g:32044)

[CO1] Cohen, D. and Orlik, P., Arrangements and local systems, Math. Res. Lett. 7 (2000), no.2-3, 299-316. MR.1764324 (2001i:57040)

[CO2] Cohen, D. and Orlik, P., Gauss-Manin connections for arrangements, I Eigenvalues, Compositio Math. 136 (2003), no. 3, 299-316. MR1977008 (2004a:32042)

[CS] Cohen, D. and Suciu, A., Characteristic varieties of arrangements, Math. Proc. Cambridge Philos. Soc. 127 (1999), 33-54. MR1692519 (2000m:32036)

[De] Deligne, P., Équations différentielles à points singuliers réguliers, Lecture Notes in Math. 163 (1970). MR0417174 (54:5232)

[ESV] Esnault, H., Schechtman, V., and Viehweg, E., Cohomology of local systems on the complement of hyperplanes, Invent. Math. 109 (1992), 557-561; Erratum 112 (1993), 447. MR1176205 (93g:32051) MR.1213111 (94b:32061)

[EFK] Etingof, P., Frenkel, I., and Kirillov, A. Jr., Lectures on representation theory and Knizhnik-Zamolodchikov equations, Math. Surveys and Monographs 58, Amer. Math. Soc., 1998. MR.1629472 (2001b:32028)

[Fa] Falk, M., Combinatorial and algebraic structure in Orlik-Solomon algebras, European J. Combin. 22 (2001), no. 5, 687-698. Mr.1845492 (2002f:52023)

[FT] Falk, M. and Terao, H., $\beta N B C$-bases for cohomology of local systems on hyperplane complements, Trans. Amer. Math. Soc. 349 (1997), no. 1, 189-202. MR1401770 (97g:52029)

[Gel] Gelfand, I., General theory of hypergeometric functions, Soviet Math. Dokl. 33 (1986), $573-577$.

[Ka] Kawahara, Y., The twisted De Rham cohomology for Basic Constructions of Hyperplane arrangements and its applications, preprint. 
[Li] Libgober, A., Characteristic varieties of algebraic curves, Applications of algebraic geometry to coding theory, physics and computation (Eilat, 2001), 215-254, NATO Sci. Ser. II Math. Phys. Chem., 36, Kluwer Acad. Publ., Dordrecht, 2001. MR.1866902 (2003e:14008)

[LY] Libgober, A. and Yuzvinsky, S., Cohomology of the Orlik-Solomon algebras and local systems, Compositio Math. 121 (2000), no. 3, 337-361. MR.1761630 (2001j:52032)

[OT] Orlik, P. and Terao, H., Arrangements of Hyperplanes, Grundlehren der mathematischen Wissenschaften 300, Springer-Verlag, (1992). MF,1217488 (94e:52014)

[OT2] - Arrangements and Hypergeometric integrals, MSJ, Mem. vol.9, Math. Soc. Japan, (2001). MR1814008 (2003a:32048)

[STV] Schechtman, V., Terao, H., and Varchenko, A., Local systems over complements of hyperplanes and the Kac-Kazhdan conditions for singular vectors, J. Pure Appl. Algebra 100 (1995), 93-102. MF 1344845 (96j:32047)

[Te] Terao, H., Moduli space of combinatorially equivalent arrangements of hyperplanes and logarithmic Gauss-Manin connections, Topology Appl. 118 (2002), 255-274. MR1877728 (2003e:32049)

[Va] Varchenko, A., Multidimensional Hypergeometric Functions and Representation Theory of Lie Algebras and Quantum Groups, Adv. Ser. Math. Phys., vol. 21, World Scientific, River Edge, NJ, 1995. MR1384760 (99i:32029)

[Yu1] Yuzvinsky, S., Cohomology of the Brieskorn-Orlik-Solomon algebras, Comm. Algebra 23 (1995), 5339-5354. MR.1363606 (97a:52023)

[Yu2] Yuzvinsky, S., Orlik-Solomon algebras in algebra and topology, (Russian) Uspekhi Mat. Nauk 56 (2001), no. 2(338), 87-166; translation in Russian Math. Surveys 56 (2001), no. 2, 293-364. MR $1859708(2002 \mathrm{i}: 14047)$

Department of Mathematics, Tokyo Metropolitan University, Minami-Ohsawa 1-1, HACHIOJI-SHI, TOKYO 192-0397, JAPAN

E-mail address: ykawa@comp.metro-u.ac.jp 